\title{
Situación del cuyamel (Joturus pichardi): pez de importancia cultural y comercial para los pobladores de la zona cultural de la Reserva del Hombre y la Biósfera del Río Plátano
}

\section{Situation of the cuyamel (Joturus pichardi Poey, 1860), fish of cultural and commercial importance for the inhabitants of the Cultural Zone of the Reserva del Hombre and the Biosfera del Río Plátano}

\begin{abstract}
RESUMEN
El área de estudio se encuentra en La Reserva del Hombre y Biosfera del Río Plátano (RHBRP), patrimonio mundial para la humanidad desde 1982 de acuerdo a la Organización de las Naciones Unidas para la Educación, la Ciencia y la Cultura (UNESCO).

El objetivo de esta investigación fue dar respuesta a la preocupación manifestada por los pobladores de la reserva sobre la disminución del cuyamel (Joturus pichardi) en la pesca artesanal. Dicha inquietud fue expresada al Programa de Adaptación al Cambio Climático en el Sector Forestal (CLIFOR) de la Cooperación Alemana y al Instituto de Conservación y Desarrollo Forestal, Áreas Protegidas y Vida Silvestre (ICF).

Por tratarse de una especie de importancia cultural y un objeto de conservación, ambas instituciones buscaron la colaboración de la Escuela de Biología de la Facultad de Ciencias/UNAH para diseñar y ejecutar el proyecto de vinculación e investigación que se presenta en este documento. Inicia una alianza estratégica entre las tres entidades para beneficio de las comunidades de la RHBRP.

Se aplicó el "Protocolo de Monitoreo de Pesca Marina y Lagunar" (López, et al, 2018), basado en el modelo analítico tradicional propuesto por Sparre y Venema (1997). Simultáneamente se aplicaron encuestas sobre la pesca de cuyamel a los pescadores locales, quie-
\end{abstract}

Ester Agar

López Irías ${ }^{1}$,

Heydi Paola

Gómez Valladares²,

Nelly Arjany

Callejas Alvarez ${ }^{3}$,

Jorge Alberto

Pérez Antúnez ${ }^{4}$,

Marcio Arnoldo

Martínez ${ }^{5}$

Rut Ester

Pinoth Arguijo6 Hans Jörg Kräuter ${ }^{7}$

\footnotetext{
Profesora universitaria y asesora, Escuela de Biología, Facultad de Ciencias, UNAH. Correo electrónico: ester.lopez@unah.edu.hn.

Licenciada en Biología, DIGEPESCA. Correo electrónico: heydi.gomez@unah.hn

${ }^{3}$ Licenciada en Biología. ICF, Técnico SIMONI, Región Biosfera del Rio Plátano Correo electrónico: argicallejas.a@gmail.com 4Licenciado en Biología, asesor, Escuela de Biología, Facultad de Ciencias, UNAH. Correo electrónico: japerez@unah.edu.hn 5Licenciado en Biología, ICF: ICF Técnico de la Regional Biosfera del Rio Plátano, oficina local de Marañones. Correo electrónico: sphyrnam@yahoo.es 'Ingeniera agrónomo, CLIFOR. Correo electrónico: rut.pinoth@giz.de ${ }^{7}$ Coordinador Equipos Regionales GIZ-GFA, CLIFOR. Correo electrónico: hkrauter.gfa@gmail.com
} 
nes indicaron la disminución de la población de peces en los últimos años debido a la sobrepesca, el uso de dinamita y la aplicación de veneno. A pesar de ser la especie central del estudio, el cuyamel (Joturus pichardi) no resultó ser una de las principales especies de captura con lo que se corroboró la percepción de la comunidad sobre la especie.

Se debe tener en cuenta que existe una pesca ilegal dirigida hacia el cuyamel, sobre todo practicada por pescadores provenientes de otras zonas del país. Es por esta razón que se recomienda continuar con los monitoreos en la pesca artesanal de las comunidades dentro de la biósfera, así como la capacitación de guarda recursos y técnicos locales para la recopilación de datos biométricos de los individuos de cuyamel capturados durante las temporadas seca y lluviosa. Por otra parte, se debe considerar las posibilidades de cultivar esta especie en su hábitat natural.

Palabras clave: cuyamel, Río Plátano, pesca artesanal.

\section{SUMMARY}

The study area is in the Reserva del Hombre y la Biosfera del Río Plátano (RHBRP), a world heritage site for humanity since 1982 according to the United Nations Educational, Scientific and Cultural Organization (UNESCO).

The objective of this research was to respond a concern expressed by the residents of the reserve regarding the decline of the Cuyamel (Joturus pichardi) in artisanal fisheries. This concern was expressed to the Program of Adaptation to Climate Change in the Forest Sector (CLIFOR) of the German Cooperation and to the Institute of Forest Conservation and Development, Protected Areas and Wildlife (ICF).

As it is a species of cultural importance and an object of conservation, both institutions searched for the collaboration from the Biology School at the Science Faculty / UNAH to design and execute the linkage and research project presented in this document.This initiative gave birth to a strategic alliance between the three entities for the benefit of the RHBRP communities.

The "Marine and Lagunar Fishing Monitoring Protocol" by López, et al, (2018) was applied, which is based on the traditional analytical model proposed by Sparre and Venema (1997). In addition to biological sampling, surveys were conducted on the fishing activities of the local fishermen, who indicated the decrease of fish population in recent years due to overfishing, the use of dynamite and the application of poison. In spite of being the central species of the study, the Cuyamel (Joturus pichardi) did not turn out to be one of the target species of capture with which the perception of the community on the species was corroborated. 
It should be borne in mind that there is an illegal fishing directed towards the Cuyamel, especially by fishermen from other parts of the country. For this reason that we recommend to continue with the monitoring of the artisanal fisheries of the communities within the biosphere, as well as the training of local resources and technicians for the collection of biometric data of individuals captured during the dry and rainy season. On the other hand, the possibilities of cultivating this species in its natural habitat should be considered.

Keywords: cuyamel, Río Plátano, artisanal fishing.

\section{Introducción}

a Reserva del Hombre y Biosfera del Río Plátano (RHBRP), es el área protegida más grande de Honduras y fue declarada patrimonio mundial por la Organización de las Naciones Unidas para la Educación, la Ciencia y la Cultura (UNESCO) en 1982. Río Plátano tiene una longitud aproximada de $100 \mathrm{~km}$ con una anchura promedio de $30 \mathrm{~m}$ y una profundidad máxima de $10 \mathrm{~m}$ durante la estación seca (Cruz, 1987). El río se interconecta con un número considerable de canales y dos lagunas cuya conexión es acentuada durante la temporada lluviosa en los meses de octubre a enero (Cruz, 1987).

Uno de los cuerpos de agua lenticos de la RHBRP más importante es la Laguna de Ibans o Ébano (Cárdenas, 2004) que está clasifi- cada como laguna, según el glosario de términos del Plan Regional de Pesca y Acuacultura Continental (PREPAC), está localizada entre los municipios de Juan Francisco Bulnes y Brus Laguna, entre los territorios indígenas y afrohondureños de Barauda, Rayaka y DIUNAT, departamento de Gracias a Dios, Honduras. Por otra parte, la Laguna de Brus, por sus características, ha sido clasificado como costera y se encuentra localizada en el Municipio de Brus, dentro del mismo departamento y en el territorio Indígena miskito de DIUNAT.

Según el PREPAC (2005), el consumo de productos pesqueros en las comunidades de la RHBRP es alto y constituye la base de la dieta alimenticia en la zona. La pesca es practicada de manera artesanal y constituye también una 
fuente de ingreso para algunos pobladores; sin embargo, es ejercida en su mayoría con fines de autoconsumo (ESNACIFOR, 2013).

Una de las especies de peces altamente apetecidos es el cuyamel (Joturus pichardi), pez catádromo perteneciente a la familia Mugilidae, que vive en ríos y lagunas costeras y su dieta se basa en el consumo de algas (cyanophytas) del género Phormidium. Al momento de reproducirse el cuyamel, realiza una migración río abajo para ovopositar en lagunas costeras, estuarios e incluso a pocos metros de la costa. Los individuos jóvenes pueden llegar a consumir de manera accidental bajas cantidades de Chirómidos y Hemípteros (insectos), al momento de remover las algas que crecen sobre las rocas (Cruz, 1987; Villalobos y Molina, 2017).

Los indígenas Miskitos y Pech de la zona han percibido, a lo largo de los años, una reducción de la pesca del cuyamel en el Río Plátano y sus afluentes, por lo que, en el año 2012, el cuyamel fue declarado como objeto de conservación para la RHBRP, por lo tanto, toda actividad comercial a gran escala dirigida hacia este pez resulta ilegal. Sin embargo, durante el verano, pescadores provenientes de las ciudades de Tocoa y La Ceiba realizan una pesca intensiva con el objeto de capturar al cuyamel y comercializarlo fuera de la reserva. Estas actividades ilegales podrían ser las responsables de la significativa reducción en la población de esta especie.

Otro factor que debe tomarse en cuenta es la actividad pesquera en las lagunas costeras; ya que el cuyamel es una especie catádroma, la presencia de pescadores artesa- nales en estos cuerpos de agua y cruces de ríos podrían comprometer su actividad reproductiva, generando impactos en sus poblaciones a mediano y largo plazo. Además, la desforestación a lo largo del margen del Río Plátano y los cambios en su caudal podrían ser responsables de alteraciones en la calidad del agua y como consecuencia afectar la ictiofauna que habita el río. Para esta especie fueron identificadas tres amenazas directas según el Plan de Conservación de la RHBRP: pesca ilegal, construcción de la represa en el Río Wampú e invasión de especies exóticas (tilapia) (Carrasco et al 2013).

El presente artículo representa el primer estudio sobre la situación de Joturus pichardi en tres sitios dentro de la RHBRP, enfocado desde la actividad de pesca.

\section{Material y métodos}

El estudio abarcó dos temporadas distintas, la primera comenzando en septiembre de 2017 y finalizando en enero 2018, posteriormente la segunda se realizó de mayo a octubre del 2018. El proyecto fue desarrollado por dos estudiantes de práctica profesional supervisada de la carrera de Biología, UNAH; cada una participó en una etapa bajo la supervisión técnica científica de una docente de la Escuela de Biología quien diseñó el protocolo de investigación. En el área de la RHBRP las estudiantes fueron directamente supervisadas por el técnico de la regional de ICF, con el cual se coordinó la socialización del proyecto y logística necesaria.

Se aplicó el "Protocolo de Monitoreo de Pesca Marina y Lagunar" (López, et al, 2018), basado en el modelo analítico tradicional 
propuesto por Sparre y Venema (1997), donde se recopila la talla y peso de las capturas pesqueras. Luego de la socialización del proyecto en las comunidades, se efectuaron muestreos biológicos en los sitios de desembarque pesquero de la Laguna de Ibans, Río Plátano y Barra de Brus Laguna, donde se esperaba a los pescadores a tempranas horas del día.

Conforme al protocolo de monitoreo, la unidad de muestreo biológico es la embarcación seleccionada de manera aleatoria al momento de su arribo de la faena pesquera. El muestreo biológico incluye datos generales donde destacan: lugar de faena, arte aplicado, distancia para llegar a la zona, duración de la actividad entre otros. Una vez obtenida esta información se le solicitaba al encargado de la embarcación autorización para muestrear el producto.

Datos biométricos como longitud de horquilla (LH) y peso fueron re- copilados utilizando un ictiometro de madera y una balanza analítica, mientras que los sitios de muestreo se georeferenciaban (Figura 1). La distancia entre cada comunidad dificultaba el desarrollo de los monitoreos, por ejemplo, la gira entre la comunidad de Río Plátano, ubicada justo en la barra del río, y Las Marías Pech rio arriba requería aproximadamente de nueve horas de viaje en pipante (embarcación de madera con fondo plano, impulsada con motor usada en ríos y lagunas).

La identificación taxonómica se realizó haciendo uso del libro "Peces de las aguas continentales de Costa Rica" de William Bussing publicado en 1998, la "Guía para la identificación de especies para fines de pesca: centro y este del Atlántico" de la Organización de las Naciones Unidas para la Alimentación y la Agricultura (FAO) publicado en 2002, y la base de datos digital fishbase.org.

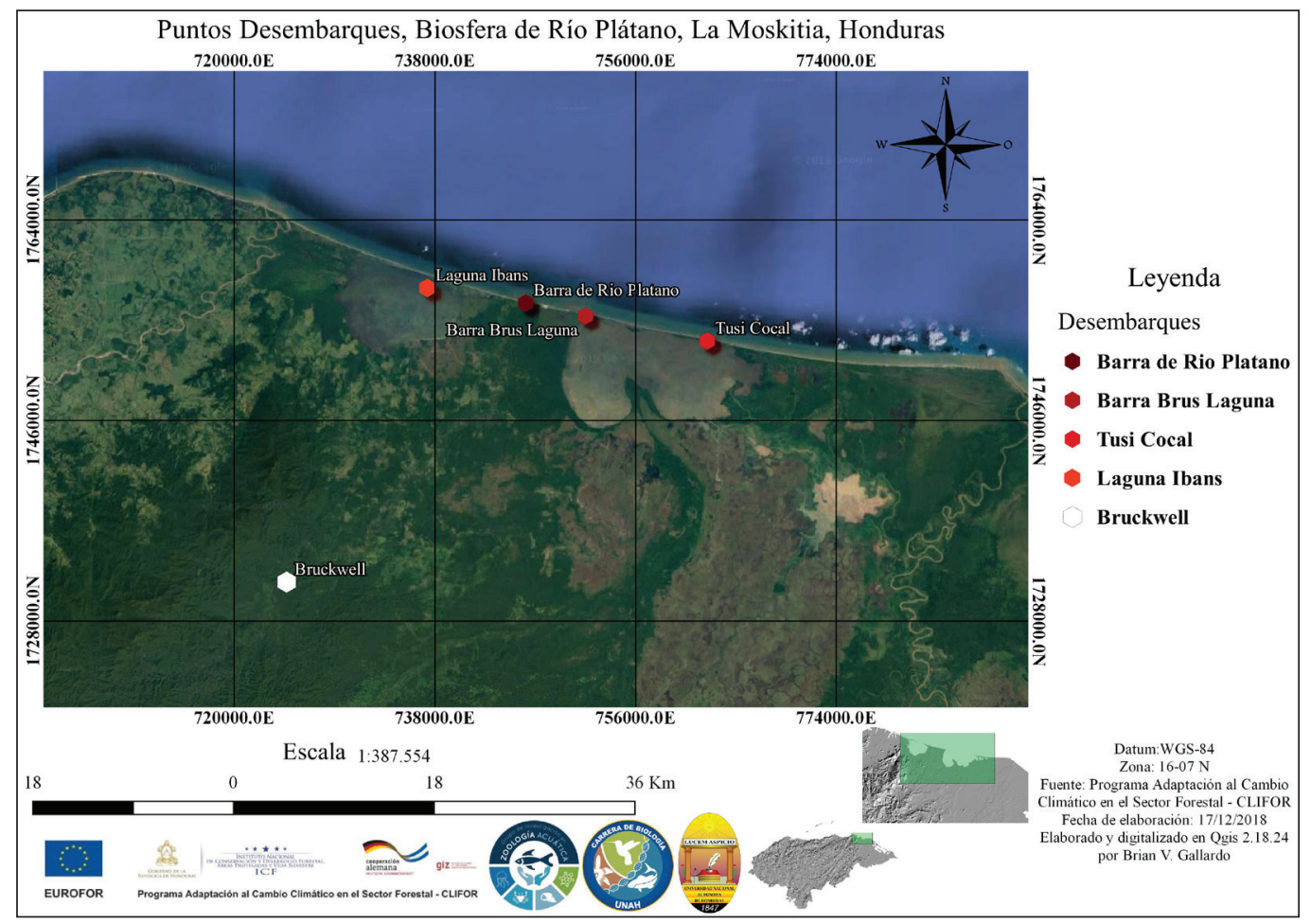

Figura No.1. Puntos de muestreo de Joturus pichardi en la RHBRP. Mapa elaborado por Brian V. Gallardo, estudiante de la carrera de Biología. 


\section{Resultados}

El principal logro es contribuir a resolver la inquietud manifestada por parte de los pobladores sobre la disminución poblacional del cuyamel (Joturus pichardi) en la pesca artesanal de La Reserva del Hombre y Biosfera del Río Plátano (RHBRP). Gracias al aporte en el conocimiento de esta especie, el Instituto de Conservación Forestal Áreas Protegida y Vida Silvestre (ICF) está desarrollando estrategias para la conservación del cuyamel, dirigiendo sus esfuerzos en áreas prioritarias.

Es aquí donde la ciencia corrobora la percepción que tienen las comunidades sobre sus recursos pesqueros, importantes no solo para su alimentación sino también para su cultura. Al respecto el presente estudio evidencia que existe una disminución en la población del cuyamel.

Al mismo tiempo este estudio generó la plataforma para establecer lazos de cooperación entre el Programa de Adaptación al Cambio Climático en el Sector Forestal (CLIFOR) de la Cooperación Alemana, el Instituto de Conservación y Desarrollo Forestal, Áreas Protegidas y Vida Silvestre (ICF) y la Escuela de Biología de la Facultad de Ciencias de la UNAH.

Esta alianza estratégica, complementó las fortalezas de las tres entidades participantes, donde el ICF con presencia institucional en la reserva aportó a este proyecto el permiso de investigación, logística y acompañamiento en el área durante todo el estudio además de ser la institución encargada de aplicar la normativa y ejecutar el plan de manejo. La Cooperación Alemana (CLIFOR), financió toda la investigación y contribuyó en todo momento con la logística y el correcto desarrollo del proyecto.

En tanto la UNAH aportó el diseño del protocolo de investigación y la ejecución del proyecto a cargo de dos estudiantes de práctica profesional supervisada, previo a la obtención de su licenciatura en Biología y bajo la supervisión permanente de una docente. Las estudiantes vivieron en la reserva durante toda su práctica, lo que representó para ellas un crecimiento a nivel personal y profesional. Heidy Gómez participó en la primera etapa del proyecto y Nelly Callejas durante la segunda, ambas coautoras de esta publicación.

Lo anterior brindó la oportunidad de compartir conocimientos y experiencias entre las estudiantes practicantes y los pescadores de la RHBRP. Este intercambio de información ayudará a crear herramientas para la sostenibilidad de los recursos naturales lo que beneficia directamente a las comunidades en la Moskitia.

Resalta la publicación de una nota informativa incluida en el "Resumen de Tesis y Pasantías Profesionales" elaborado por el Programa Adaptación al Cambio Climático en el Sector Forestal - CLIFOR en el año 2019. 


\section{Características de la pesca artesanal}

El trasmallo es el arte de pesca para la captura del cuyamel en las lagunas, mientras que en el río se realiza con arpón por buceo libre ó apnea (Figura 2.a), específicamente en la época seca cuando hay visibilidad en el agua, en la época lluviosa la pesca se hace con red.

Los pescadores de la Barra de Río Plátano señalaron que la mejor época de pesca de cuyamel es durante el mes de noviembre, es- pecíficamente los días 20, 21 y 22. Para estas fechas algunos miskitos de la comunidad de Río Plátano utilizan trasmallos (Figura 2.b) en el río con el objetivo de capturar cuyamel.

El tipo de embarcación empleada en la pesca en el río es el "pipante" (Figura 2.a), construido de madera con fondo plano, impulsada con motor. En las lagunas se usa más la lancha para la pesca.

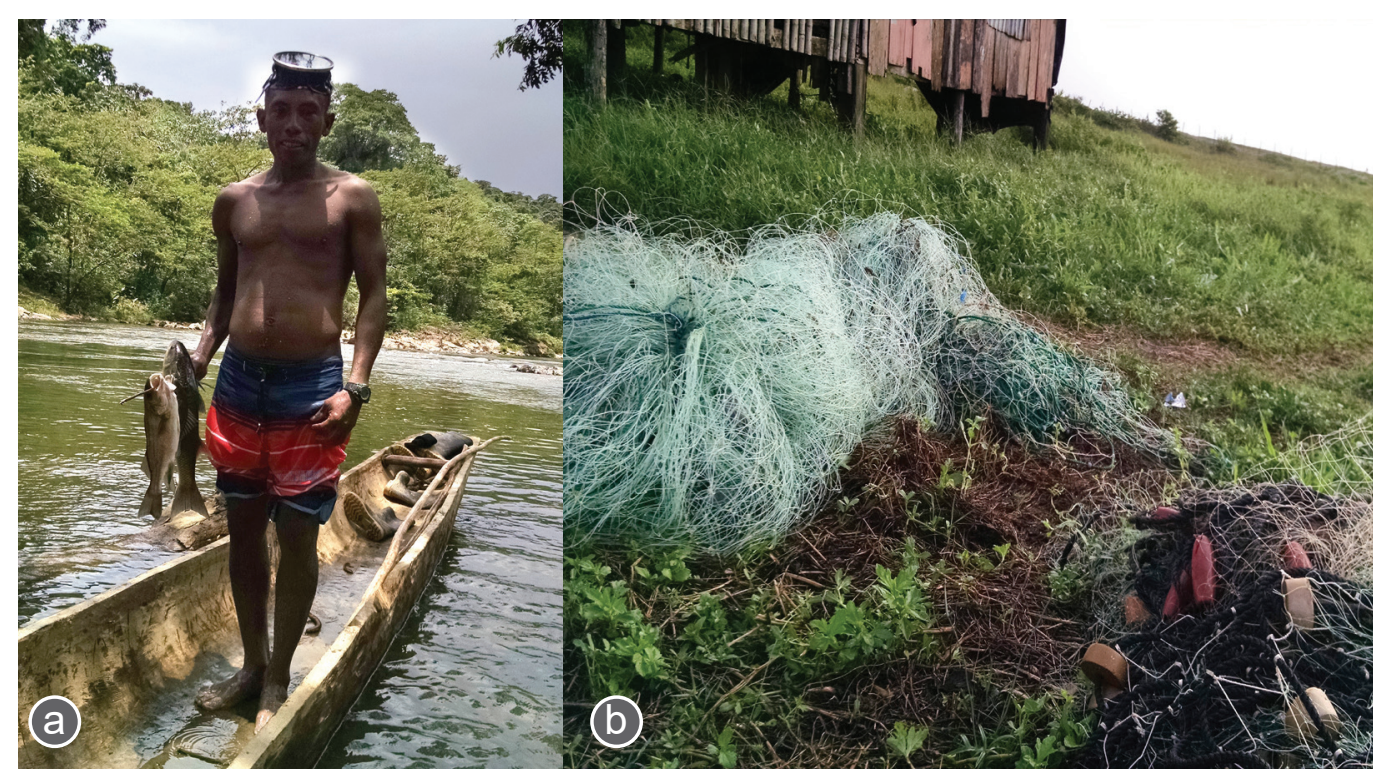

Figura No. 2. a. Pescador miskito capturando Joturus pichardi con arpón en Río Plátano. b. artesanal en la zona de la RHBRP Trasmallo utilizado para la pesca.

\section{Entrevistas dirigidas a pescadores sobre cuyamel (Joturus pichardi).}

Uno de los resultados más destacables fue la información recopilada directamente de los pescadores, sobre el conocimiento que tienen del cuyamel. Lo anterior es indispensable para elaborar un documento que sirva como herramienta para la sostenibilidad de los recursos en la zona y sobre todo a la pesca dirigida al cuyamel.

Se aplicaron 65 encuestas en las que se abordaban temas que incluían tallas actuales y anteriores en la pesca de cuyamel (Joturus pichardi) y diferencias entre libras capturadas, precios y su comercio. Los pescadores señalaron a los compradores procedentes del departamento de Olancho y la ciudad de Tocoa como la principal vía de comercio del cuyamel, aunque cabe resaltar la presencia de un comprador de origen guatemalte$\mathrm{co}$, quien adquiría el producto a un valor de L100.00 (precio por pez) en Brus Laguna (Figura 3). 
Figura No. 3. Origen de los compradores de cuyamel en la RHBRP

\section{Origen de los compradores}

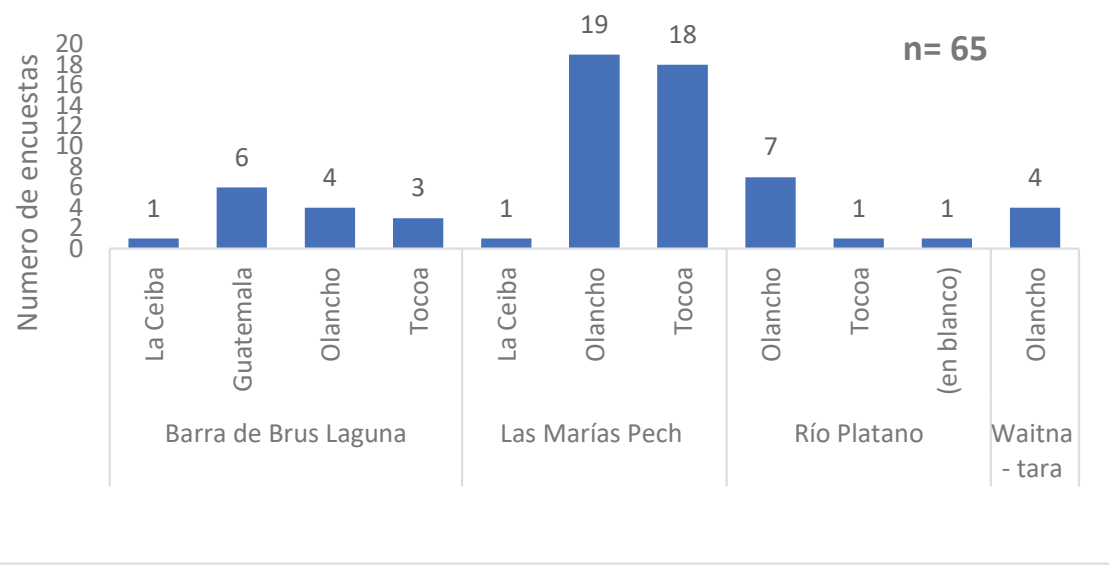

Figura No 4.

Percepción sobre la abundancia del cuyamel en la pesca artesanal de las comunidades.

De acuerdo a la percepción de los pescadores, el cuyamel no es una especie abundante en la pesca de Brus Laguna y la comunidad de Río Plátano, a diferencia de las Marías Pech, donde el $28 \%$ de pescadores lo consideran abundante pero solamente durante la temporada entre marzo y abril (Figura 4).

Como se puede apreciar en la figura 5 , en la comunidad de Las Marías Pech se dan los mayores precios de venta por individuo de cuyamel (300 Lempiras). Cabe resaltar que la venta de cuyamel es mayor durante el verano, época del año donde aumenta el número de compradores de cuyamel procedentes de otros sitios, incluso desde Guatemala.
El cuyamel es tradicionalmente uno de los peces más vistos y capturadas en Río Plátano, sin embargo, según lo recopilado a lo largo del estudio, tanto en muestreos biológicos como en entrevistas dirigidas a pescadores artesanales de la especie, las poblaciones han sufrido un declive en los últimos años. En la figura 6 , se aprecia que la mayoría de los pescadores entrevistados señalan que la disminución en tallas de la población de cuyamel ha ocurrido aproximadamente durante los últimos cinco años (Figura 7) lo que coincide con el aumento de pesca por parte de ladinos procedentes de otros municipios y/o departamentos. 


\section{Precios por libra de cuyamel}

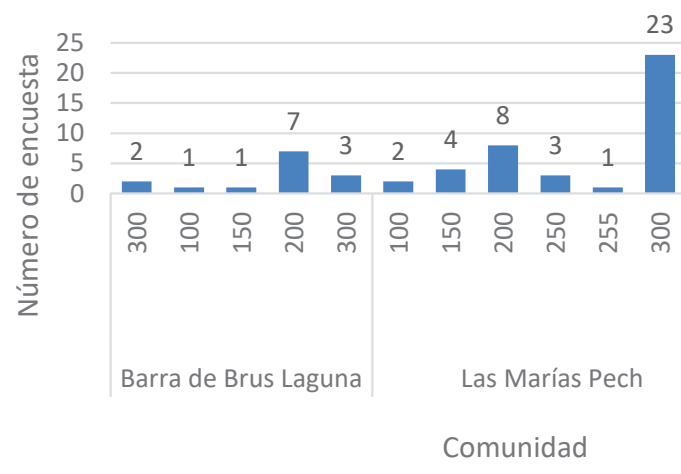

Figura No 5.

Precios de venta de cuyamel por libra en las comunidades.

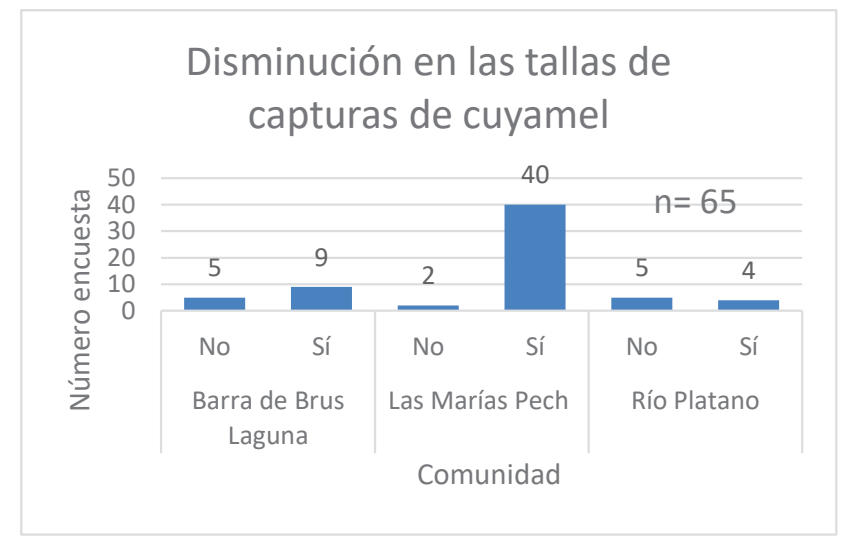

Período en que se redujo la población de cuyamel

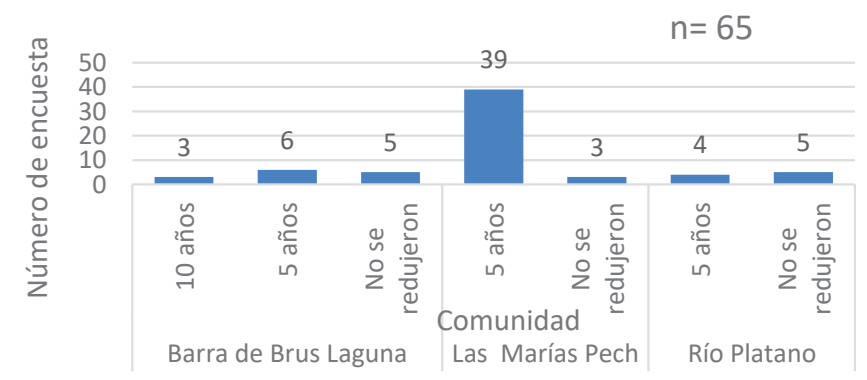

Figura No 6 y 7. Disminución en las tallas de captura de cuyamel y periodo de tiempo en el que ocurrió la reducción de capturas.

Los pescadores afirman que antes de la reducción en la población de cuyamel, las capturas de este pez eran numerosas, de hasta 100 individuos con tallas mayores a 50 cm; sin embargo, en la actualidad también se aprecia una reducción considerable en la talla del pez (Figura 8 y 9).

Según las encuestas aplicadas a pescadores de la Barra de Brus
Laguna y en la zona de Las Marías Pech, las tallas de cuyamel se redujeron en los últimos años. Ellos afirman que antes de esta reducción se capturaba tallas mayores de $50 \mathrm{~cm}$ en comparación con las tallas actuales que oscilan entre 30- 49cm LH (Figura 10), por otra parte, el número de capturas de cuyamel también ha disminuido (Figura 11).

\section{Aspectos biológicos pesqueros}

Se realizó un total de 85 muestreos biológicos dirigidos a la pesca artesanal en la Laguna de lbans, en el Río Plátano y Brus Laguna, sin embargo, el cuyamel (Joturus pichardi) sólo fue capturado en la Laguna de Brus, debido a que no ingresa a la Laguna de lbans, posiblemente como resultado de elevadas concentraciones de salinidad (Carrasco, 2004). El porcentaje de cuyamel en las capturas de los muestreos biológicos fue de $0.02 \%$ (Figura 12), ocupando la décima cuarta posición. 
Situación del Cuyamel Joturus pichardi: pez de importancia cultural y comercial para los pobladores de la Zona Cultural de la Reserva del Hombre y la Biosfera del Río Plátano

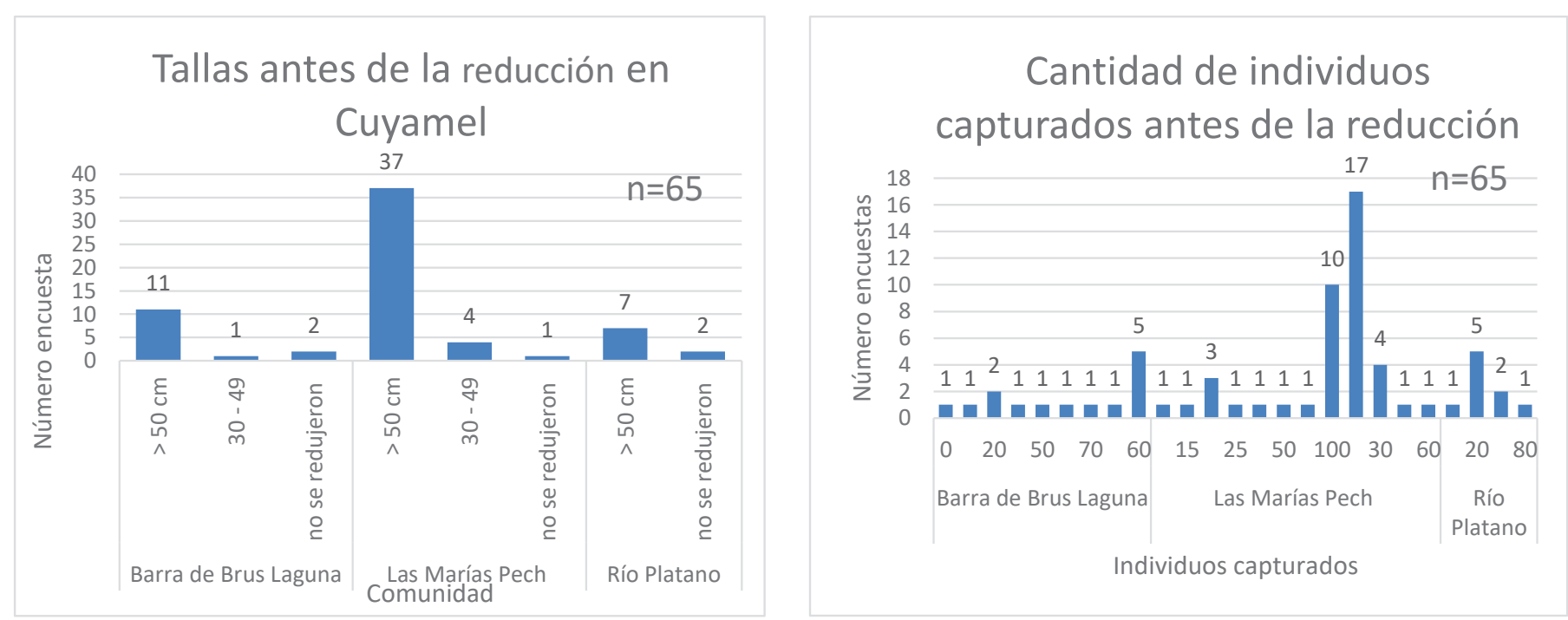

Figura No 8 y 9 . Tallas de capturas de cuyamel antes de la reducción y cantidad de individuos capturados
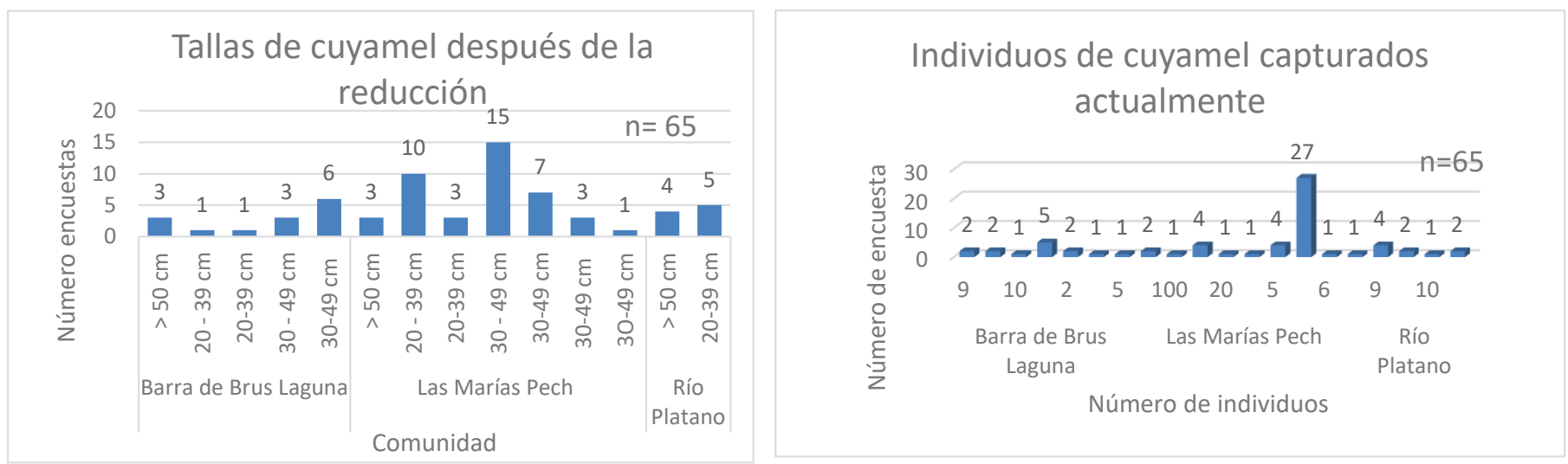

Figura No 10 y 11. Tallas de captura de cuyamel después de la reducción poblacional e individuos capturados actualmente.

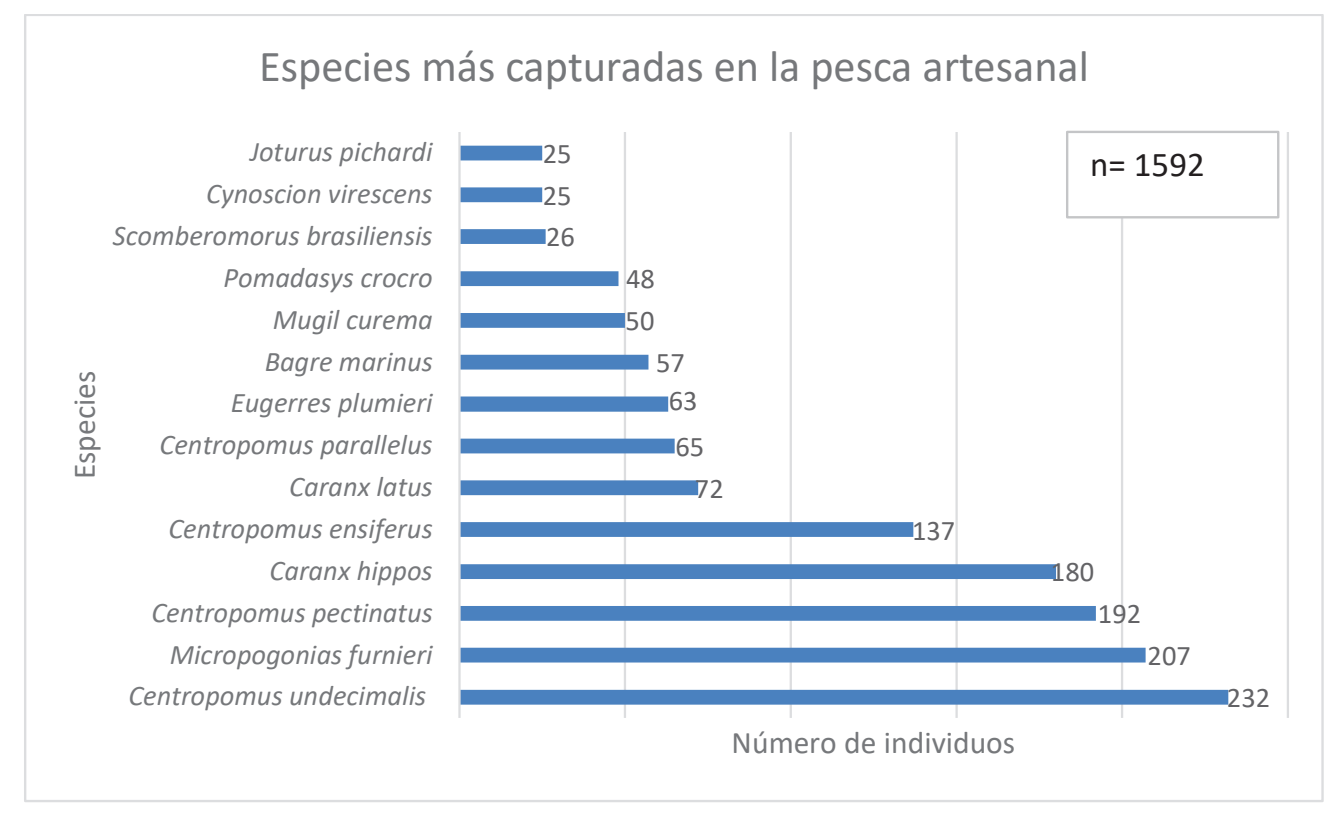

Figura No. 12. Posición del Cuyamel (Joturus pichardi) en la captura de la RHBRP. 
Durante todo el estudio fueron muestreados un total de 25 individuos de Joturus pichardi, 22 provenían de la Laguna de Brus mientras que los 3 restantes procedían de Las Marías Pech en el lugar conocido como Bruckwell (Figura 12). Los 10 individuos sexados presentaron madurez sexual con una talla promedio de $60 \mathrm{~cm}$ de longitud de horquilla (LH), siendo la talla de 41 $\mathrm{cm}$ de $\mathrm{LH}$ en hembras reportada por Cruz (1987) como la de primera maduración.

Las hembras fueron más comunes que los machos en los muestreos (Figura 13); mientras que para los individuos procesados no fue posible obtener datos de sexo ni estadío gonadal. La mayoría de los muestreos se realizaron en organismos procesados (salados) que fueron encontrados en su totalidad en la Barra de Río Plátano y Barra de Brus Laguna. Cabe mencionar que este tipo de conservación no permite obtener las biometrías requeridas por el protocolo de monitoreo.

Sin embargo, debido a la baja cantidad de datos, las biometrías de los individuos salados de cuyamel son presentadas en la tabla 1 , donde las cifras de peso en gra-

Tabla 1. Listado de cuyamel (Joturus pichardi) muestreados en la Barra de Brus Laguna y Río Plátano, específicamente en los barrios de la comunidad de Las Marías Pech.

\begin{tabular}{|c|c|c|c|c|c|}
\hline $\mathbf{N}^{\mathbf{0}}$ & Longitud $\mathbf{( c m )}$ & Peso $\mathbf{( g )}$ & Sexo & Estadio gonadal & Estado \\
\hline 1 & $\mathbf{4 3}$ & 1043 & $\mathrm{H}$ & $\mathrm{E} \mathrm{IV}$ & Fresco \\
\hline 2 & $\mathbf{6 0}$ & 1361 & $\mathrm{H}$ & $\mathrm{E} \mathrm{IV}$ & Fresco \\
\hline 3 & $\mathbf{7 3}$ & 1814 & $\mathrm{M}$ & $\mathrm{E} \mathrm{II}$ & Fresco \\
\hline 4 & $\mathbf{5 5}$ & 1588 & $\mathrm{M}$ & $\mathrm{E} \mathrm{II}$ & Fresco \\
\hline 5 & $\mathbf{5 6}$ & 1406 & $\mathrm{M}$ & $\mathrm{E} \mathrm{II}$ & Fresco \\
\hline 6 & $\mathbf{6 0}$ & 2267 & $\mathrm{H}$ & $\mathrm{E} \mathrm{IV}$ & Fresco \\
\hline 7 & $\mathbf{5 6}$ & 2723 & $\mathrm{H}$ & $\mathrm{E} \mathrm{IV}$ & Fresco \\
\hline 8 & $\mathbf{4 3}$ & 1135 & $\mathrm{H}$ & $\mathrm{E} \mathrm{II}$ & Fresco \\
\hline 9 & $\mathbf{4 7}$ & 1249 & $\mathrm{H}$ & $\mathrm{E} \mathrm{II}$ & Fresco \\
\hline 10 & $\mathbf{2 6}$ & 246 & $\mathrm{M}$ & $\mathrm{E} \mathrm{I}$ & Fresco \\
\hline 11 & $\mathbf{6 1}$ & 1814 & $\mathrm{I}$ & 0 & Salado \\
\hline 12 & $\mathbf{3 9}$ & 908 & $\mathrm{I}$ & 0 & Salado \\
\hline 13 & $\mathbf{5 1}$ & 1587 & $\mathrm{I}$ & 0 & Salado \\
\hline 14 & $\mathbf{3 8}$ & 862 & $\mathrm{I}$ & 0 & Salado \\
\hline 15 & $\mathbf{4 4}$ & 1088 & $\mathrm{I}$ & 0 & Salado \\
\hline 16 & $\mathbf{4 3}$ & 1134 & $\mathrm{I}$ & 0 & Salado \\
\hline 17 & $\mathbf{4 3}$ & 1043 & $\mathrm{I}$ & 0 & Salado \\
\hline 18 & $\mathbf{4 0}$ & 952 & $\mathrm{I}$ & 0 & Salado \\
\hline 19 & $\mathbf{4 1}$ & 907 & $\mathrm{I}$ & 0 & Salado \\
\hline 20 & $\mathbf{4 3}$ & 998 & $\mathrm{I}$ & 0 & Salado \\
\hline 21 & $\mathbf{4 4}$ & 1043 & $\mathrm{I}$ & 0 & Salado \\
\hline 22 & $\mathbf{4 1}$ & 952 & $\mathrm{I}$ & 0 & Salado \\
\hline 23 & $\mathbf{3 8}$ & 907 & $\mathrm{I}$ & 0 & Salado \\
\hline 24 & $\mathbf{4 2}$ & 910 & $\mathrm{I}$ & 0 & Salado \\
\hline 25 & $\mathbf{4 6}$ & 1350 & $\mathrm{I}$ & 0 & Salado \\
\hline
\end{tabular}

H: hembra, M: mancho, I: sexo indeterminado, E IV: estadio gonadal IV, E II: estadio gonadal II, El: estadio gonadal I, $\mathbf{0}$ : sin determinar estado sexual.

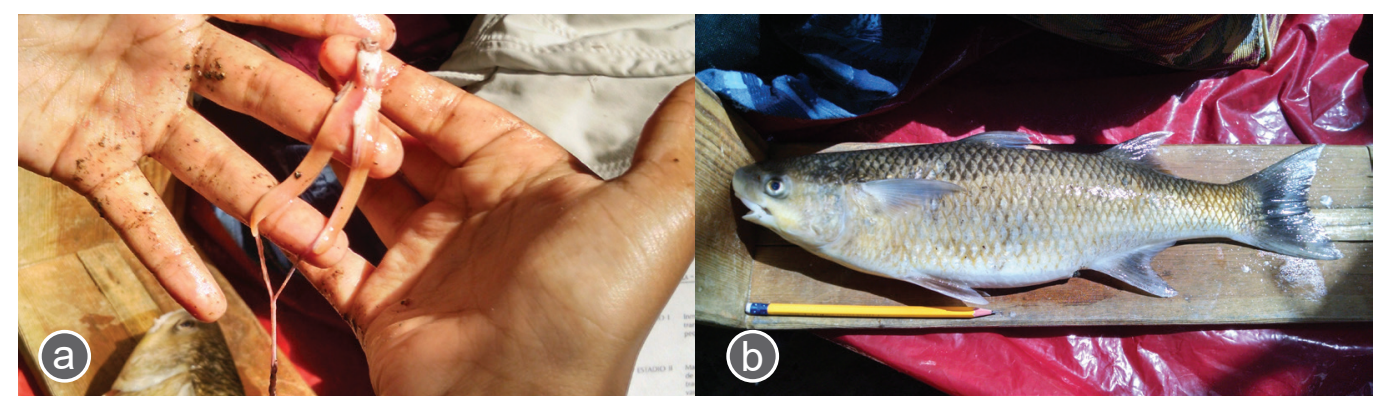

Figura No. 13. a. Estadio gonadal E-II en hembra de Joturus pichardi (E II- Estadio gonadal II). b. ejemplar de J. pichardi capturado en la zona de Bruckwell en Las Marías Pech. 
mos de los individuos en estado fresco representan un peso entero y los individuos en estado salado corresponden a un peso eviscerado del producto.

Los muestreos en la barra de Brus Laguna fueron realizados a embarcaciones de pescadores permanentes, tanto ladinos como miskitos y garífunas, quienes mostraron su colaboración la mayor parte del tiempo, pese a las dificultades climáticas de la pesca durante la primera etapa del estudio (octubre a diciembre de 2017), cuando las lluvias son más intensas y se desata la respuesta migratoria del cuyamel (Cruz et al, 1987).

Sin embargo, el número de individuos de cuyamel fue bajo. Duran- te el año 2018 no se pudo observar individuos de cuyamel en esta zona durante el mes de octubre, fenómeno que se adjudica a la ausencia de lluvia para este periodo de tiempo. Otro punto georeferenciado fue Tusi Cocal, muestreado en 2017, donde se reportaron dos individuos de cuyamel, uno salado y una hembra en estadío IV.

De manera complementaria, datos de parámetros físico-químicos fueron recopilados durante la época lluviosa y parte de la época seca. Según la información de ESNACIFOR, (2013) y el presente estudio, Río Plátano presenta parámetros de calidad de agua dentro del rango habitable para el cuyamel (Joturus pichardi).

Tabla 2. Parámetros de calidad de agua en el Río Plátano, Laguna de Ibans y Barra de Brus Laguna.

\begin{tabular}{|l|c|c|c|c|c|}
\hline Áreas & Temperatura $\left({ }^{\circ} \mathbf{C}\right)$ & $\mathbf{p H}$ & Alcalinidad (mg/L) & Conductividad (Ms) & Fuente \\
\hline Rio Plátano & 26.7 & 6 & 40 & 45 & Datos de estudio \\
\hline Laguna Ibans & 31.3 & 5 & 0 & 10.96 & Datos de estudio \\
\hline Laguna Brus & 26 & 5 & --- & --- & Datos de estudio \\
\hline Rangos RHBRP & $22-27$ & $6-7.24$ & $17-63$ & $20-139$ & $($ ESNACIFOR, 2013) \\
\hline
\end{tabular}

\section{Discusión}

El cuyamel (Joturus pichardi) es muy apreciado por su carne y debido a lo difícil de su pesca, se ha optado por aplicar métodos ilegales de captura como la dinamita lo que destruye los ecosistemas y pone en peligro el futuro de la especie (Cruz, 1987). Molina (2009), afirma que estas prácticas no solo son realizadas en Honduras sino también en Costa Rica, lo que sumado a la sobrepesca, ha provocado una disminución de esta especie en el territorio costarricense. La presión sobre la población de cuyamel también se registra en Colombia, donde se presume una disminución debido al deterioro y cambio ambiental, ya que esta especie está condicionada por la época con mayores valores de pluviosidad además de la sobre pesca (Eslava y Díaz, 2011). 
La mayoría de los individuos encontrados en los muestreos biológicos fueron hembras en estadio gonadal IV encontradas en la primera etapa de la investigación (octubre 2017-enero 2018) donde se reportaron condiciones fluviales para la temporada de desove del cuyamel, esto concuerda con lo reportado por Molina (2009) quien indicó la existencia de una incidencia mucho mayor de hembras maduras en estadio IV en el Río Sarapiquí en Costa Rica, durante el mes de octubre.

La disminución de Joturus pichardi se puede ver reflejada no solo en las encuestas aplicadas a los pescadores artesanales de la especie, sino también en los muestreos biológicos que se realizaron. Cruz (1988) reportó que, por noche, en un muestreo biológico y utilizando trasmallo, se podían capturar hasta 50 individuos entre machos y hembras sexualmente maduros a $300 \mathrm{~m}$ de una desembocadura del Rio Plátano. Al comparar los resultados de Cruz (1988) con los datos del presente estudio, se observa una disminución en la población de cuyamel.

La Laguna de Ibans es uno de los sitios de la RHBRP donde se presumía que podría encontrarse el Joturus pichardi pero después de las dos etapas de la investigación no se logró muestrear ningún individuo. Tradicionalmente, y por conocimiento de los pescadores de la Laguna de lbans, el cuyamel no ha sido una especie comúnmente capturada en esta zona. Según el Inventario de los cuerpos de agua de Honduras con énfasis en la pesca y acuicultura de 2005, cuando el Río Plátano se desborda, incor- pora parte de sus aguas a la laguna a través del angosto segmento de sabana y mangle que lo separa, pero la Laguna de Ibans recibe aportes mayormente de tres diferentes riachuelos, uno de estos y el considerado más importante es Wapniyari con limitadas condiciones para soportar una población importante de cuyamel, por lo que se presume que esta sería la razón por la que el cuyamel no llegue a desovar en la laguna.

La laguna de lbans también recibe las aguas del Río Sico (antes llamado Rio Tinto o Negro) y es precisamente este río el que impide el ingreso de las cuñas de agua salada hasta la laguna, por lo que se mantiene dulce durante todo el año. Es este fenómeno otro factor importante para entender porque el cuyamel no desove en este cuerpo de agua, ya que la especie prefiere el agua salobre para la puesta de sus huevos (AFE-COHDEFOR, 2002). Lo anterior es relevante porque permite al ICF dirigir los esfuerzos de conservación a zonas prioritarias donde existan condiciones para sostener las poblaciones de cuyamel.

Los datos de temperatura recopilados durante el estudio (promedio de $26.7^{\circ} \mathrm{C}$ ) concuerdan con los presentados por ESNACIFOR, (2013) que fluctúan entre $22-27^{\circ} \mathrm{C}$, valores dentro del rango habitable para cuyamel según el mismo documento. Sin embargo, Bussing, (1998) y Eslava (2009) indican que las temperaturas ideales para el hábitat del cuyamel oscilan entre $22-25^{\circ} \mathrm{C}$. Lo anterior es importante, si se contempla las posibilidades del cultivo de cuyamel en la reserva. 


\section{Conclusiones}

En un principio se sostenía la idea que el cuyamel estaría entre las especies más capturadas y que sería más relevante para la dieta de los indígenas, no obstante, las encuestas señalan que cuando se captura es una fuente de ingreso de mucha importancia, sobre todo después de ser procesado lo que aumenta su precio en el mercado.

Es importante mencionar que el estudio no abarcó los meses de marzo y abril, periodo señalado como el de mayor pesca de cuyamel, por lo que se recomienda hacer una evaluación puntual con muestreos biológicos durante estos meses a futuro. Además, se debe tomar en cuenta que solamente se pudieron evaluar 10 individuos frescos enteros, por lo que debe definirse la estrategia para que los muestreos biológicos se hagan en la cuenca alta del Río Plátano, antes del salado del pescado. Lo anteriormente recomendado implica un mayor costo en logística.

De igual manera, es recomendable dar seguimiento a los monitoreos de la pesca artesanal, enfatizando en las comunidades aledañas a las lagunas costeras por tratarse de zonas de pesca muy diversas y priorizar la capacitación de los guarda recursos y técnicos locales para la recopilación de datos biométricos de las especies capturadas en las diferentes estaciones del año. El incremento en el número de datos permitiría obtener tasas de mortalidad para determinar el estado de la población de cuyamel. La Unión Internacional para la Conservación de la Naturaleza (UICN) en el 2018 reporta al cuyamel como una especie no evaluada, dentro de la lista roja de las especies amenazadas.

Los robalos y roncos son las principales especies de la pesca en Las Marías Pech, pero estas que no tienen un valor comercial para los pescadores ya que ellos no comercializan estos productos. Sin embargo, estos peces si representan una fuente de alimento para las familias, por lo que dicha actividad de captura es realizada por hombres, mujeres y niños. En otras partes de Honduras las distintas especies de robalo tienen un alto valor comercial y su presencia en la zona representa un potencial para la comercialización del producto de manera responsable.

\section{Agradecimientos}

A todas las personas que ayudaron a la realización de esta investigación especialmente las comunidades de Las Marías Pech, Laguna de lbans y Barra de Brus Laguna y a los pescadores que siempre apoyaron y tuvieron la disposición de ayudar. Un agradecimiento espe- cial a ICF, especialmente a la oficina local de Palacios por su cooperación durante todo el proceso. Asimismo, deseamos agradecer al Programa de Adaptación al Cambio Climático en el Sector Forestal por el apoyo y la confianza brindada. 


\section{Bibliografía}

AFE / COHDEFOR. 1996. Plan Operativo Refugio de Vida Silvestre y Pesca Artesanal El Jicarito.35p.

Bussing, W.A., 1998. Peces de las aguas continentales de Costa Rica. 2nd Ed. San José Costa Rica: Editorial de la Universidad de Costa Rica. $468 \mathrm{p}$.

Carrasco, J.C., Portillo, H., Estuardo, S. y Lara, K. 2013. Plan de Conservación de la Reserva del Hombre y la Biósfera del Río Plátano: Basado en Análisis de Amenazas, Situación y del Impacto del Cambio Climático, y Definición de Metas y Estrategias. ICF y USAID ProParque. $86 \mathrm{p}$.

Cárdenas P., 2004. Datos limnológicos, 2004. Proporcionados por el Ing. Pablo Cárdenas - Jefe de la Unidad Ambiental Municipal.

Cruz, G. 1987. Reproductive biology and feeding habitat of Cuyamel, Joturus pichardi and Tepemechín, Agonostomus monticola (Pisces; Mugilidae) from Río Plátano, Mosquitia, Honduras. Bulletin of Marine Science. 40: 63-72.

Cruz, G. 1988. Joturus pichardi (Pisces; Mugilidae) sexualmente maduros capturados en Laguna de Brus y en la desembocadura del Río Plátano, Honduras. Rev. Biología Tropical.

Eslava, P. 2009. Estimación del rendimiento y valor nutricional del besote Joturus pichardi Poey, 1860 (Pisces: Mugilidae). Rev. MVZ Cordoba. 14 (1).

Eslava, P. y Días, R. 2011. Reproducción de Joturus pichardi y Agonostomus montícola (Mugiliformes: Mugilidae) en ríos de la Sierra Nevada de Santa Marta, Colombia. Rev. Bio. Trop. 59 (4): 1717-1728.

Escuela Nacional de Ciencias Forestales (ESNACIFOR). 2013. Plan de Manejo Reserva del Hombre y Biósfera del Río Plátano (2013 - 2025) DAP, ICF. Región Biósfera de Río Plátano.

IUCN, 2018. IUCN Red List of Threatened Species. Versión 2018-2. Descargado en Noviembre 19 de 2018.

López, E., Sierra, L., y Pérez, J. 2018. Protocolo de Monitoreo de Pesca Marina y Lagunar.

Molina, A. 2009. Monitoreo del estado de madurez gonadal del pez bobo, Joturus pichardi (Poey 1860) en el rio Sarapiquí. Rev. Vida Sector Electricidad. (5) p. 25-29.

Plan Regional de Pesca y Acuacultura Continental (PREPAC), 2005. Inventario de los cuerpos de agua de Honduras con énfasis en la pesca y acuicultura. p. 65-70, 226-235.

Villalobos, J. y Molina, A. 2017. Migración y estado de maduración del Pez Bobo (Joturus pichardi) en la Cuenca del Río Sarapiquí, Heredia; Costa Rica. Instituto Costarricense de Electricidad. Unidad de Manejo de Cuencas. 9 p. 